http://jmscr.igmpublication.org/home/ ISSN (e)-2347-176x ISSN (p) 2455-0450

crossref DOI: https://dx.doi.org/10.18535/jmscr/v8i12.14

Journal Of Medical Science And Clinical Research

IGM Publication

An Official Publication of IGM Publication

\title{
Mortality among COVID-19 Patients- A Retrospective Study
}

\author{
Authors \\ Dr Amandeep Singh ${ }^{1}$, Dr Pankaj Jorwal ${ }^{*}$, Dr Upendra Baitha ${ }^{3}$, Dr Manish Soneja ${ }^{4}$, \\ Dr Ashutosh Biswas, MD $^{5}$ \\ ${ }^{1}$ Senior Resident, ${ }^{2}$ Associate Professor, ${ }^{3}$ Assistant Professor, \\ ${ }^{4}$ Additional Professor, ${ }^{5}$ Professor \\ Department of Medicine, All India Institute of Medical Sciences (AIIMS), New Delhi \\ *Corresponding Author \\ Dr Pankaj Jorwal
}

\begin{abstract}
Objectives: Since December 2019, COVID-19 is engulfing the whole world day by day. It has a wide range of clinical manifestations which are evolving daily. We reviewed the factors associated with mortality among admitted COVID-19 patients by analysing their clinical profile and lab parameters.

Methods: Case records of 50 COVID-19 cases admitted to a medicine unit at AIIMS, New Delhi from April to mid-June were analysed retrospectively after applying inclusion and exclusion criteria. The course of disease was compared among survivors and non survivors.

Results: Among 50 subjects, 86\% were below 60 years of age and $14 \%$ were above 60, mortality was higher in elderly age group and was statistically significant $(p<0.05)$. Most patients were symptomatic; fever and dyspnoea being common presenting complain seen in $62 \%$ of patients followed by cough, myalgia, sore throat, diarrhoea and rash. Among patients who presented with all three symptoms at presentation (fever, cough \& dyspnoea), $43 \%$ succumbed to disease. Looking at severity, 38\% were mild, $42 \%$ were moderate and $20 \%$ were severe cases at admission. The mortality was highest in severe group (90\%). Prior co morbidities were seen in 60\% cases. Hypertension (24\%) was the commonest followed by diabetes (18\%) and CKD (6\%). $66.7 \%$ of patients with CKD died. Among non-survivors, 37.5\% were anaemic and $72.7 \%$ had leucocytosis ( $p<0.05) .53 .8 \%$ patients with raised urea and $36.3 \%$ patients with raised creatinine also died.
\end{abstract}

Conclusion: Older age, anaemia, leucocytosis and deranged renal function at the presentation are significantly associated with mortality among admitted COVID-19 patients.

Keywords: COVID-19 Disease, Risk Factors, Mortality.

\section{Introduction}

At the end of year 2019, an outbreak of acute respiratory infection occurred in the Wuhan province of China that has wobbled the world. The virus is from the family of corona viruses and is labelled as SARS-Cov-2, because of its high similarity to SARS-CoV, which led to epidemic in
2002-2003. The disease has been labelled as COVID-19. The virus has spread worldwide leading to declaration of pandemic by WHO on $11^{\text {th }}$ March 2020. ${ }^{[1]}$ Currently by 21 August 22, 925, 540 COVID-19 Cases along with 798,237 deaths occurred in 215 countries in the world. The United States of America is having the maximum 
number of cases till date i.e. 5,757,888 followed by Brazil and India respectively ${ }^{[2]}$. First case of COVID-19 in India was detected in Kerala, on 30 January 2020. At present here are about 692028 active cases in India, 2158946 cured and with 54849 deaths. $^{\text {[3] }}$

Human to Human transmission is the main route of transmission of SARS-Cov-2. Both symptomatic as well as those who are asymptomatic can transmit the virus thus isolating the patient is the best way to prevent it from spreading. The respiratory droplets (5-10 micrometre) generated from cough or sneeze transmit the virus from COVID positive patient to those who are in close contact. Individuals who are in pre-symptomatic stage lead to $80 \%$ of transmission $^{[4]}$. The incubation period is 14 days. Most common symptoms of COVID-19 are fever, difficulty in breathing and dry cough. Other less common presenting features are diarrhoea, fatigue, myalgia, anorexia and vomiting ${ }^{[5]}$. Many patients becomes critical due to multi-organ dysfunction and Acute Respiratory Distress Syndrome (ARDS). Still after 5 months of declaration of pandemic, the complete clinical picture of the COVID-19 is unclear ${ }^{[6]}$. Thus, we planned a retrospective study to review the clinical as well as lab profile of the COVID-19 patients and analyse the significant factors associated with mortality.

\section{Methodology}

\section{Study Design and Participants}

This was a retrospective study wherein case records of confirmed COVID-19 adult patients admitted in Unit III of Department of Medicine at AIIMS New Delhi between $1^{\text {st }}$ April to $15^{\text {th }}$ June were analysed. The study was approved by institutes' ethical committee. Patients were followed up till discharged or death. Diagnosis was done as per the ICMR guidelines for COVID19. Patients with incomplete records were excluded from the study.

\section{Data Collection \& Study Variables}

The medical records of patients were obtained from hospital case records. All data was captured in a pre-designed Performa, filled in a excel sheet and analysed. Outcome of the patients, i.e. survivor or non-survivor were the two major groups in which the subjects were divided for analysis.

\section{Statistical Analysis}

Statistical analysis was carried out using STATA v15.1, STATA Corp. LLC, TX, and USA. For testing of hypothesis, two tailed tests were considered and $\mathrm{p}$ value of $<0.05$ was considered to be as statistically significant. Descriptive analysis was carried out first to summarise the study population. Data distribution was checked by normal probability plot and Kolmogorov Simonov test. A continuous variable with no extreme values (normally distributed) was given by mean +- standard deviation. Continuous variables with extreme values (skewed) were given by Median (IQR) and number (\%) was given for qualitative variables. For the comparison of two groups student's t-test was performed if it had normal distribution otherwise Mann Whitney U-test was used. Difference in proportion was assessed by using Pearson Chi- square test or Fisher's exact test.

\section{Results}

Data of 50 COVID-19 positive patients was analysed after applying inclusion and exclusion criteria. Mean age of the patients was $45.54 \pm 14.4$ yrs. Three groups were made on the basis of age i.e. Group 1 ( $<45$ yrs.) included $48 \%$ patients, Group 2 (45-60yrs) included $38 \%$ patients and Group3 (> 60 yrs.) included $14 \%$ patients as shown in table 1 . In group $3,57.1 \%$ of patients could not survive whereas in group 1 and 2 only $20 \%$ and $10 \%$ were non-survivors respectively, depicting higher mortality in the elderly population $(\mathrm{p}<0.05)$. Overall $62 \%$ patients were males and $38 \%$ were females, $22.5 \%$ males and $21.0 \%$ female patients did not survive but the difference was not statistically significant. 
About $99 \%$ of the patients were symptomatic at presentation, with fever and dyspnoea being the most common symptoms, seen in $62 \%$ of patients followed by cough (58\%), myalgia (28\%), anorexia (12\%), sore throat $(10 \%)$, diarrhoea $(4 \%)$, headache (4\%) and $\operatorname{rash}(2 \%)$ as shown in table 2. $44 \%$ of the patients presented with fever and cough both, $40 \%$, 36\% with fever and dyspnoea combined and $32 \%$ patients presented with fever, dyspnoea and cough all three combined and maximum death $(43.7 \%)$ was seen among the patients who presented with all three symptoms. Anorexia was seen in $12 \%$ of patients among which $50 \%$ were non-survivors. $10 \%$ of total patients presented with sore throat, in which no mortality was seen.

As per the latest guidelines of ICMR, COVID-19 patients can be divided into three groups i.e. mild, moderate and severe. Mild patients are defined as those with uncomplicated upper respiratory tract infection, have mild symptoms such as fever, cough, sore throat, nasal congestion without dyspnoea. Moderate patients are those who present with clinical features of dyspnoea and/or hypoxia, fever, cough, including $\mathrm{SpO} 2$ (range 90$94 \%$ ) on room air, respiratory rate more or equal to 24 per minute. All those patients with clinical sign of pneumonia along with $\mathrm{SpO} 2<90 \%$ on room air or respiratory rate $>30$ are to be considered as severe cases ${ }^{[7]}$. In our study, $38 \%$ patients were mild, $42 \%$ were moderate and $20 \%$ patients were severe at presentation. Among these, 90\% patients of severe category died whereas the mortality was less in mild and moderate category of patients.

In our study $60 \%$ of the total patients had comorbidities whereas death was seen in only $23.3 \%$ and majority of the patients $(76.7 \%)$ survived. Hypertension was the most common comorbidity seen in $24 \%$ of total patients, followed by diabetes (18\%), Chronic Kidney Disease $(6 \%)$, past history of pulmonary Tuberculosis and Asthma (4\% each), COPD (2\%) and treated case of malignancy $(2 \%)$.
$66.7 \%$ of the patients with chronic Kidney disease expired whereas only $11 \%$ of diabetes patients and $25 \%$ of patients with hypertension died.

As per the World Health Organization, Anaemia is defined as haemoglobin of less than $13 \mathrm{~g} / \mathrm{dL}$ in male and $12 \mathrm{~g} / \mathrm{dL}$ in non-pregnant females ${ }^{[8]}$. In our patients, $48 \%$ of the total patients had anaemia in which $37.5 \%$ of anaemic patients died. In more analysis, $20 \%$ of total patients had haemoglobin $<10 \mathrm{~g} / \mathrm{dl}$ and $50 \%$ of these patients were non-survivors. The result was statistically significant. $(\mathrm{p}<.05)$

As per our laboratory parameters reports, Total Leucocyte Count is considered normal in the range of 4000-110000. In our study, $72 \%$ of total patients had normal leukocyte count, $6 \%$ had leukopenia and $22 \%$ patients had leucocytosis. $72.7 \%$ of patients with leucocytosis were nonsurvivors whereas there was no death observed in patients with leukopenia. The result was statistically significant. $(\mathrm{p}<0.05)$. In our study $70 \%$ patients had normal platelet count,28\% showed thrombocytopenia and only $2 \%$ had raised platelet count.22.8 \% of patients with normal platelets and $21.1 \%$ of patients with thrombocytopenia died whereas no death was seen in patients with thrombocytosis. The result was not statistically significant. $(\mathrm{p}=0.81)$

In our study, $26 \%$ of the total patients had raised serum urea levels and $53.8 \%$ of these patients couldn't survive. The results were statistically significant $(\mathrm{p}<0.05) .44 \%$ of the total population had raised serum creatinine at the time of presentation and $36.3 \%$ patients among this died. The result was also statistically significant $(p<0.05) .90 \%$ of the total patients had normal bilirubin levels whereas only $10 \%$ of the remaining patients had raised bilirubin levels. 
Table 1 Age and sex characteristics of COVID-19 patients

\begin{tabular}{|cl|c|c|c|}
\hline CHARACTERISTIC & $\begin{array}{c}\text { TOTAL(N= 50) } \\
(\%)\end{array}$ & NON-SURVIVORS (\%) & SURVIVORS (\%) \\
\hline AGE & \multicolumn{3}{|c|}{} \\
\hline$\bullet$ & $<45$ & 48 & 20 & 80 \\
\hline$\bullet$ & $45-60$ & 38 & 10.5 & 89.5 \\
\hline$\bullet \quad>60$ & 14 & 57.1 & 42.9 \\
\hline SEX & & & & 77.5 \\
\hline$\bullet$ & Male & 62 & 22.5 & 79 \\
\hline$\bullet$ & Female & 38 & 21.0 & \\
\hline
\end{tabular}

Table 2 Clinical Profile, Severity and Co-morbidities in COVID-19 patients

\begin{tabular}{|c|c|c|c|}
\hline CHARACTERISTIC & $\begin{array}{c}\text { TOTAL, } \\
\mathrm{N}=50 \mathrm{In} \%(100)\end{array}$ & NON -SURVIVORS (\%) & SURVIVORS (\%) \\
\hline \multicolumn{4}{|l|}{ SYMPTOMS } \\
\hline - Fever & 62 & 25.8 & 74.2 \\
\hline - $\quad$ Dyspnoea & 62 & 32.2 & 67.8 \\
\hline - Cough & 58 & 31 & 69 \\
\hline - $\quad$ Fever +Cough & 44 & 36.3 & 63.7 \\
\hline - $\quad$ Dyspnoea + Cough & 40 & 40 & 60 \\
\hline - $\quad$ Fever +Dyspnoea & 36 & 38.8 & 61.2 \\
\hline - $\quad$ Fever +Dyspnoea+ Cough & 32 & 43.7 & 56.3 \\
\hline - $\quad$ Myalgia & 28 & 21.4 & 78.5 \\
\hline - $\quad$ Anorexia & 12 & 50 & 50 \\
\hline - $\quad$ Sore throat & 10 & 0 & 100 \\
\hline - $\quad$ Headache & 4 & 0 & 100 \\
\hline - $\quad$ Diarrhoea & 4 & 50 & 50 \\
\hline - Rash & 2 & 0 & 100 \\
\hline \multicolumn{4}{|l|}{ SEVERITY } \\
\hline • $\quad$ Mild & 38 & 5.2 & 94.8 \\
\hline - $\quad$ Moderate & 42 & 4.7 & 95.3 \\
\hline - $\quad$ Severe & 20 & 90 & 10 \\
\hline \multicolumn{4}{|l|}{ COMORBIDITY } \\
\hline Total & 60 & 23.3 & 76.7 \\
\hline - $\quad$ Hypertension & 24 & 25 & 75 \\
\hline - $\quad$ Diabetes & 18 & 11.1 & 88.9 \\
\hline - $\quad \mathrm{CKD}$ & 6 & 66.7 & 33.3 \\
\hline - $\quad$ Old Pulmonary TB & 4 & 50 & 50 \\
\hline - $\quad$ Asthma & 4 & 0 & 100 \\
\hline - $\quad$ COPD & 2 & 100 & 0 \\
\hline $\begin{array}{ll}- & \text { Carcinoma Thyroid(Treated) }\end{array}$ & 2 & 0 & 100 \\
\hline
\end{tabular}

CKD (Chronic Kidney Disease), TB (Tuberculosis), COPD (Chronic Obstructive Pulmonary Disease)

Table 3 Laboratory values at the admission of COVID-19 patient

\begin{tabular}{|c|c|c|c|}
\hline CHARACTERISTICS & Total $(\mathrm{N}-\mathbf{5 0}) \%$ & NON-SURVIVORS (\%) & SURVIVORS(\% \\
\hline \multicolumn{4}{|l|}{$\mathrm{Hb}$} \\
\hline $\operatorname{Normal}(13-15 \mathrm{~g} / \mathrm{dl})$ & 52 & 7.6 & 92.4 \\
\hline $\begin{array}{l}\text { - } \quad \text { Low }(\text { Male }<13 \\
\text { Female }<12)\end{array}$ & 48 & 37.5 & 62.5 \\
\hline - $\quad$ Very $\operatorname{Low}(<10)$ & 20 & 50 & 50 \\
\hline \multicolumn{4}{|l|}{ TLC } \\
\hline Normal $(4.0-11.00) \times 10^{3} / \mu \mathrm{L}$ & 72 & 8.3 & 91.7 \\
\hline - $\quad \operatorname{Low}\left(<4.0 \times 10^{3} / \mu \mathrm{L}\right)$ & 6 & 0 & 100 \\
\hline - $\quad \operatorname{High}\left(>11 \times 10^{3} / \mu \mathrm{L}\right)$ & 22 & 72.7 & 27.3 \\
\hline \multicolumn{4}{|l|}{ PLATELET } \\
\hline - $\quad \operatorname{Normal}(150-400) \times 10^{3} / \mu \mathrm{L}$ & 70 & 22.8 & 77.2 \\
\hline - $\quad \operatorname{Low}\left(<150 \times 10^{3} / \mu \mathrm{L}\right)$ & 28 & 21.4 & 78.6 \\
\hline - $\quad \operatorname{High}\left(>400 \times 10^{3} / \mu \mathrm{L}\right)$ & 2 & 0 & 100 \\
\hline \multicolumn{4}{|l|}{ Urea } \\
\hline Normal(10-40)mg/dL & 74 & 10.8 & 89.2 \\
\hline - $\quad \operatorname{High}(>40 \mathrm{mg} / \mathrm{dL})$ & 26 & 53.8 & 46.1 \\
\hline \multicolumn{4}{|l|}{ CREATININE } \\
\hline - $\quad \cdot \operatorname{Normal}(0.50-1.00) \mathrm{mg} / \mathrm{dL}$ & 56 & 10.7 & 89.3 \\
\hline • $\quad \cdot \operatorname{High}(>1.0 \mathrm{mg} / \mathrm{dL})$ & 44 & 36.3 & 63.7 \\
\hline \multicolumn{4}{|l|}{ BILIRUBIN } \\
\hline - $\quad \operatorname{Normal}(0.2-1.20) \mathrm{mg} / \mathrm{dL}$ & 90 & 24.4 & 75.6 \\
\hline - $\quad \cdot \operatorname{High}(>1.20 \mathrm{mg} / \mathrm{dL})$ & 10 & 0 & 100 \\
\hline
\end{tabular}

Hb (Hemoglobin), TLC (Total Leucocyte Count), 


\section{Discussion}

COVID-19 pandemic is ongoing and it seems, will remain with us for a considerable amount of time. There are a limited number of studies describing clinical as well as laboratory finding of COVID-19 patients along with their mortality. There were about 50 patients whose data was analysed in present study out of which 39 were survivors and 11 non-survivors. In our study age was significantly associated with mortality in COVID-19 patients. Senior citizens or patients who were more than 60 year of age had a high risk of mortality. More than $50 \%$ of our patients with age above 60 succumbed to illness. Similar results were seen in the study done by Hongdou Li et.al in which among 76 non-survivors, most patients were older with an average age of 71.47 and death was rare in younger population. ${ }^{[9]}$

In present study majority of the patients were symptomatic with fever and dyspnoea being the most common symptoms followed by cough, myalgia, anorexia, sore throat, diarrhoea, headache and rash. We observed that patients who presented with combined three symptoms of fever, cough and dyspnoea had high mortality. More than $40 \%$ of these patients did not survive although the results were not statistically significant. A study done by Wang et.al. on 107 COVID patients also showed that fever was the most common symptom present in 104 patients, fatigue was $2^{\text {nd }}$ most common, followed by cough and dyspnoea. Majority of non survivors had dyspnoea as the presenting symptom ${ }^{[5]}$. Thus, dyspnoea at presentation is an indicator of poor prognosis, though study on large no of patients will be required to validate it.

In our study the majority of the population presented with mild to moderate illness. $38 \%$ patients were mild, $42 \%$ patients were moderate and $20 \%$ patients were severe. Only 5.2 and 4.7 $\%$ of the patients were non-survivors in mild and moderate groups respectively. All patients who died in mild and moderate groups had age of more than 65 years along with comorbidity. Thus age is a non-modifiable factor and has a great effect on the mortality. About $90 \%$ of patients who presented with severe illness died. J. Zhang et.al. in their study on 663 COVID-19 divided patients in four groups., where $0.5 \%$ of the patients were diagnosed with mild COVID-19, while $37.8 \%$, $47.5 \%$, and $14.2 \%$ were in moderate, severe, and critical conditions, respectively. In this study also patients presenting with severe illness had high mortality but was not statistically significant ${ }^{[10]}$.

Hypertension was the most common comorbidity followed by diabetes and chronic kidney disease. In our study, $66.7 \%$ of patients who had prior comorbidity of Chronic Kidney Disease died whereas only $25 \%$ of those with hypertension and $11 \%$ with diabetes died. In a study done by Rong-Hui Du et.al. on 179 patients of COVID, $32.4 \%$ of patients had hypertension as the most common comorbidity followed by diabetes in $18.4 \%$.Chronic Renal insufficiency was seen in only $2.2 \%$ of the patients. Hypertension was significantly associated with high mortality in contradiction to present study. Similar to our study Rong-Hui Du et.al. also showed no significant difference in survivors and non survivors groups among diabetic patients ${ }^{[11]}$.

In present study, low haemoglobin was significantly associated with mortality in COVID19 patients. $37.5 \%$ of patients with anaemia were died in comparison to normal haemoglobin group in which only $7.5 \%$ of patients died. The difference was statistically significant. Huang et al. have reported reduction in haemoglobin levels in $38.2 \%$ of patients admitted for COVID-19 ${ }^{[12]}$. Another study by L Wang et al. also reported low haemoglobin level in $19.23 \%$ of the study population admitted to hospital ${ }^{[13]}$. Petek Eylul Taneri et.al. did a systematic review and metaanalysis on anaemia and iron metabolism in COVID-19 and compared haemoglobin levels in moderate to severe cases where it was seen that haemoglobin was low in severe cases of COVID19, but the difference was not statistically significant. ${ }^{[14]}$

Among patients with leucocytosis, $72.7 \%$ of the patients were non- survivors, the result was 
statistically significant. A retrospective cohort study by Fei ZhouTing et.al. had also shown that out of 191 patients more than $60 \%$ of patients who had leucocytosis were non-survivors, the result was also statistically significant. Thus raised leucocyte at the presentation predicts a worse outcome $^{[15]}$.

In our study, only $28 \%$ of the total patients had thrombocytopenia out of which $21.4 \%$ of patients were non survivors but the result was not statistically significant. The result was contradictory to the similar studies by Fei ZhouTing et.al.in which there was significant thrombocytopenia among non-survivor patients compared to survivors ${ }^{[15]}$. The study by Wang, D et.al. also shown that thrombocytopenia was significantly present in non-survivor groups than in survivors group. ${ }^{[5]}$

More than $50 \%$ of the patients with raised serum urea and $36.3 \%$ of patients with raised serum creatinine were non-survivors and the difference was statistically significant. A study by Lang Wang et.al. in which 339 patients were analysed, Serum urea and creatinine were higher in nonsurvivor group than in survivor group and results were statistically significant. ${ }^{[13]}$

In our study $90 \%$ of patients had normal bilirubin and in only $10 \%$ of patients, bilirubin was high. No mortality was seen in patients with raised bilirubin levels. Rong-Hui Du et.al. Also showed that there was no significant difference in bilirubin among survivors and non survivors. ${ }^{[1]}$

\section{Conclusion}

Age is a non-modifiable risk factor and strongly associated with mortality in COVID-19 cases. Anaemia, raised TLC and deranged renal function at the presentation are associated with poor outcome in patients and thus need to be taken into account while triaging patients.

Conflict of interest: There is no conflict of interest for this research by the authors.

Funding statement: This research is not funded.

\section{References}

1. Koichi Yuki, Miho Fujiogi, Sophia Koutsogiannaki, COVID-19 pathophysiology: A review, Clin Immunol. 2020 Jun; 215: 108427

2. https://www.worldometers.info/coronaviru s/?utm_campaign=homeAdUOA?Si(Acces sed on 21 August 2020)

3. https://www.mohfw.gov.in/(Accessed on 21 August 2020)

4. Li Q, Guan X, Wu P, et al. Early transmission dynamics in Wuhan, China, of novel coronavirus-infected pneumonia, NEngl J Med. Published Online First: 29 January 2020. doi: https://doi.org/10.1056/NEJMoa2001316

5. Wang, D., Yin, Y., Hu, C. et al. Clinical course and outcome of 107 patients infected with the novel coronavirus, SARS-CoV-2, discharged from two hospitals in Wuhan, China. Crit Care24, 188

https://doi.org/10.1186/s13054-02002895-6

6. Huang C, Wang Y, Li X, et al. Clinical features of patients infected with 2019 novel coronavirus in Wuhan, China. Lancet. Published Online First: 24 January 2020. doi: https://doi.org/10.1016/S01406736(20)30183-5

7. https://www.mohfw.gov.in/pdf/ClinicalMa nagementProtocolforCOVID19.pdf( Accessed on 6 August 2020)

8. https://www.who.int/vmnis/indicators/hae moglobin.pdf Accessed on 6 th August 2020)

9. Hongdou Li, Shuang Wang, Fan Zhong, Wuyin Bao, Yipeng Li, Lei Liu, Hongyan Wang, Yungang He, Age-Dependent Risks of Incidence and Mortality of COVID-19 in Hubei Province and Other Parts of China, Front Med (Lausanne) 2020; 7: 190.

10. J. Zhang, X. Wang, X. Jia, J. Li, K. Hu, G. Chen, J. Wei, Z. Gong, C. Zhou, H. Yu, 
M. Yu, H. Lei, F. Cheng, B. Zhang, Y. Xu, G. Wang, W. Dong Risk factors for disease severity, unimprovement, and mortality in COVID-19 patients in Wuhan, China Clin Microbiol Infect. 2020 Jun; 26(6): 767-772. Published online 2020 Apr 15.doi: 10.1016/j.cmi.2020.04.01

11. Du R-H, Liang L-R, Yang C-Q, et al. Predictors of Mortality for Patients with COVID-19 Pneumonia Caused by SARSCoV-2: A Prospective Cohort Study. Eur Respir J 2020; in press (https://doi.org/10.1183/13993003.005242020).

12. Huang Y, Tu M, Wang S, Chen S, Zhou $\mathrm{W}$, Chen D, et al. Clinical characteristics of laboratory confirmed positive cases of SARS-CoV-2 infection in Wuhan, China: A retrospective single center analysis. Travel Med Infect Dis. 2020:101606.

13. Wang L, Duan Y, Zhang W, Liang J, Xu J, Zhang Y, et al. Epidemiologic and Clinical Characteristics of 26 Cases of COVID-19 Arising from Patient-to-Patient Transmission in Liaocheng, China. Clin Epidemiol. 2020;12:387-91.

14. Petek Eylul Taneri, Sergio Alejandro Gómez-Ochoa, Erand Llanaj, Peter Francis Raguindin, Lyda Z. Rojas, Beatrice Minder Wyssmann, Doris KoppHeim,Wolf E. Hautz, Michele F. Eisenga, Oscar H. Franco , Marija Glisic, Taulant Muka, Anemia and iron metabolism in COVID-19 : A systematic review and meta-analysis

(doi:https://doi.org/10.1101/2020.06.04.20 122267)
15. Fei Zhou Ting Yu Ronghui Du Guohui Fan Ying Liu Zhibo Liu Jie Xiang Yeming Wang Bin Song Xiaoying Gu Lulu Guan Yuan Wei Hui Li Xudong Wu Jiuyang Xu Shengjin Tu Yi Zhang Hua Chen Bin Cao Clinical course and risk factors for mortality of adult in patients with COVID19 in Wuhan, China: a retrospective cohort study Lancet 2020; 395: 1054-62. 